\title{
ATRIBUTOS DE IDENTIDAD Y VALORACIÓN DE UNA BEBIDA TRADICIONAL MEXICANA $^{37}$
}

\author{
Anastacio Espeje $^{a^{*}}$, Ariadna Barrera ${ }^{a}$, Luis Ledesma $^{a}{ }$, Herany Ledesma ${ }^{a}$ \\ a'Universidad Autónoma Chapingo, Texcoco, Estado de México, México (aespejelg@chapingo.mx).
}

El pulque es una bebida ancestral única en México, que ha despertado el interés de diversas disciplinas científicas. Esta intrínsecamente ligado a la tradición e identidad en México, sin embargo, en los últimos años se ha mostrado un descenso en el consumo, lo que evidencia una escasa valoración por los consumidores y el desplazamiento de esta, por otras bebidas. El objetivo de esta investigación fue identificar atributos de identidad y valoración en una muestra de 222 consumidores de pulque en México. Se diseño una encuesta con el apoyo de la herramienta Google forms la cual contenía, variables sociodemográficas, atributos del producto y aseveraciones relacionadas con la disposición a pagar. Para medir la identidad se aplicó un análisis conceptual y para la valoración se empleó valoración contingente. Los resultados indican que sí existe comunalidad con respecto a la tradición e identidad, de tal manera que los consumidores sí asocian el producto con la identidad y tradición, mientras que la mayor valoración y disposición a pagar estuvo relacionada con atributos como denominación de origen, producido por pequeños productores y producido de manera sustentable. Se concluye que es un producto identitario y que los consumidores valoran el producto por atributos vinculados al origen.

Palabras clave: significados de compra, alimento tradicional, disposición a pagar, valorización

\section{Introducción}

El maguey pulquero se desarrolla en casi toda la altiplanicie mexicana, incluyendo regiones áridas y semiáridas de los estados de Hidalgo, Puebla, Tlaxcala, México, Querétaro, San Luis Potosí, entre otros. Sus principales especies son: A. salmiana, A. americana y A. mapisaga, las que a su vez presentan gran número de variedades cultivadas (Gómez, 2011). El maguey es un recurso natural de gran importancia en México, desde el punto de vista económico, social y agroecológico. El pulque definido en la NMX-V-0371972 como la bebida que se obtiene por fermentación alcohólica de aguamiel (substrato fermentable) obtenida del maguey pulquero, no clarificada, de color blanco, ácida y de aspecto viscoso es una bebida ancestral, única en México con gran arraigo y tradición.

Los alimentos tradicionales son elementos importantes de la cultura humana, la identidad y el patrimonio; Guerrero et al. (2009) define a los alimentos tradicionales como productos de un área local determinada producidos de manera tradicional, con propiedades sensoriales específicas. La Comisión Europea (2006) indica que para considerarse a un producto como "tradicional", este debe tener el uso comprobado en el mercado comunitario durante un período de al menos 25 años y que muestre la transmisión de su uso entre generaciones. La producción de los alimentos tradicionales se basa en prácticas clásicas de fabricación, a menudo con baja competitividad, eficiencia e inversión en investigación y desarrollo.

Más del 95\% de la producción de pulque se comercializa en pulquerías y a granel en envases plásticos de refresco, garrafas o en cualquier envase que tenga a la mano el productor y el comprador; tomando en cuenta lo anterior se sobreentiende que el pulque no tiene algún valor agregado más que el de preparar de vez en cuando algún "curado" (pulque mezclado con diferentes frutas o semillas), lo que aumenta el precio dependiendo el costo de la fruta o semilla seleccionada. Sin embargo, han surgido algunas agroindustrias que se dedican al proceso de enlatado de pulque, con el objetivo principal del generar apertura comercial en el extranjero.

\section{Objetivo}

Identificar atributos de identidad y valoración en una muestra de consumidores de pulque en México mediante la aplicación de encuestas.

\section{Metodología}

Se realizó un análisis conceptual, Guerrero et al (2009), estimando el índice de diversidad y rareza. Se formaron diez campos semánticos; posteriormente se realizó una prueba de k proporciones con un valor de confianza del 95\% para conocer si existen diferencias significativas de los campos semánticos en cada

\footnotetext{
${ }^{37}$ Proyecto 20079-C-68 financiado con fondos de la Dirección General de Investigación y Posgrado de la Universidad Autónoma Chapingo.
} 
categoría geográfica y de las categorías geográficas por cada campo semántico. Se aplicó un Análisis de Componentes Principales (ACP) para la diferenciación de los sectores geográficos mediante la conceptualización otorgada a la palabra "pulque". Se calculó la disposición a pagar, Jauregui (2019), basada en 11 consideraciones, con base en los porcentajes declarados; se aplicó un análisis de K proporciones para determinar diferencias significativas entre los conceptos. Se generaron variables dependientes binomiales y se aplicó una regresión logística con el 90\% de confianza utilizando variables independientes cuantitativas y cualitativas; posteriormente se seleccionaron aquellas variables independientes que resultaran significativas y se aplicó una repetición de la regresión logística para cada disposición a pagar evaluada.

\section{Resultados}

En el análisis conceptual, se asociaron 645 palabras con la palabra pulque en las cuatro zonas geográficas resultantes: oriente EdoMex (1), resto EdoMex (2), resto del país (3), extranjero (4); al aplicar el análisis se aprecia que existe diferencia significativa entre las zonas para el índice de diversidad (ID); para el índice de rareza (IR) se generaron dos grupos existiendo diferencia significativa entre la zona 4 y el resto. Lo anterior se muestra en el cuadro 1.

\section{Cuadro 1. Categorización de la palabra "pulque"}

\begin{tabular}{c|ccccc} 
ZONA & PT & $\begin{array}{c}\text { PALABRAS } \\
\text { DIFERENTES }\end{array}$ & HÁPAX & ID (\%) & IR (\%) \\
\hline $\mathbf{1}$ & 375 & 92 & 43 & $25 \mathbf{a}$ & $47 \mathbf{a}$ \\
$\mathbf{2}$ & 138 & 49 & 26 & $36 \mathbf{a b}$ & $53 \mathbf{a}$ \\
$\mathbf{3}$ & 125 & 63 & 35 & $50 \mathbf{b}$ & $56 \mathbf{a}$ \\
$\mathbf{4}$ & 7 & 6 & 5 & $86 \mathbf{c}$ & $83 \mathbf{~ b}$
\end{tabular}

Las proporciones de las categorías formadas en la asociación libre de palabras para "pulque" se presentan en el cuadro 2. Existe diferencia entre las categorías creadas entre cada una de las regiones. Lo tradicional es el elemento con mayor significado para los consumidores entrevistados lo cual refleja la percepción de los mexicanos sobre esta bebida. En la parte identitaria si bien los significados que le otorgan son débiles, podría deberse a que por muchos años las empresas cerveceras industriales han realizado campañas de desprestigio hacia esta bebida para favorecer el consumo de cerveza y es posible que durante varias décadas hayan logrado desarticular el significado de identidad sobre el pulque, sin embargo, durante la última década se ha intentado promover y rescatar esta bebida tradicional y emblemática de México.

Cuadro 2. Significados de compra, asociados a la palabra "pulque"

\begin{tabular}{lccc}
\multicolumn{1}{c}{ CATEGORÍA } & $\mathbf{1}$ & $\mathbf{2}$ & $\mathbf{3}$ \\
\hline RURALIDAD & $41 \mathrm{bc}$ & $18 \mathrm{ab}$ & $12 \mathrm{ab}$ \\
NACIONALISMO E IDENTIDAD & $\mathbf{1 6} \mathbf{~ a b}$ & $\mathbf{6 ~ a b}$ & $\mathbf{6 ~ a b}$ \\
ATRIBUTOS DE CALIDAD & $34 \mathrm{bc}$ & $16 \mathrm{ab}$ & $21 \mathrm{~b}$ \\
PROCESO & $48 \mathrm{~cd}$ & $23 \mathrm{~b}$ & $14 \mathrm{ab}$ \\
HEDONISMO & $49 \mathrm{~cd}$ & $16 \mathrm{ab}$ & $12 \mathrm{ab}$ \\
PERCEPCIÓN AL CONSUMIR & $27 \mathrm{abc}$ & $13 \mathrm{ab}$ & $15 \mathrm{ab}$ \\
TRADICIONAL & $\mathbf{8 5 ~ \mathbf { d }}$ & $\mathbf{2 8} \mathbf{b}$ & $\mathbf{2 1 ~ b}$ \\
ALIMENTOS & $51 \mathrm{~cd}$ & $16 \mathrm{ab}$ & $12 \mathrm{ab}$ \\
SITIOS DE CONSUMO & $5 \mathrm{a}$ & $1 \mathrm{a}$ & $0 \mathrm{a}$ \\
CONVIVENCIA & $19 \mathrm{abc}$ & $1 \mathrm{a}$ & $12 \mathrm{ab}$
\end{tabular}

Las categorías para la zona 1 se clasificaron en cuatro grupos, de los cuales, las categorías "alimentos" y "tradición" obtuvieron la mayor proporción; la conceptualización del pulque en la zona oriente del estado de México (1) se basa en los aspectos tangibles e intangibles del producto; lo anterior implica un mejor posicionamiento comercial del pulque dado que las personas basan el consumo de esta bebida tanto en aspectos tecnológicos como en aspectos afectivos y simbólicos. En la zona 2, las categorías de "tradición" y "proceso" obtuvieron mayor proporción mientras que en la zona 3, fueron "tradición" y "atributos de 
calidad". El grafico 1 muestra la relación entre las categorías formadas a partir de las palabras evocadas de "pulque" y la zona geográfica (1: zona oriente EdoMex, 2: resto EdoMex, 3: Resto del país), los dos primeros factores permiten explicar el $97.5 \%$ de la variabilidad total, el factor uno explicó el 85.62\% y el factor dos explicó el $11.53 \%$ de la variabilidad de los datos.

\section{Gráfico 1. Análisis de componentes principales de la conceptualización del pulque.}

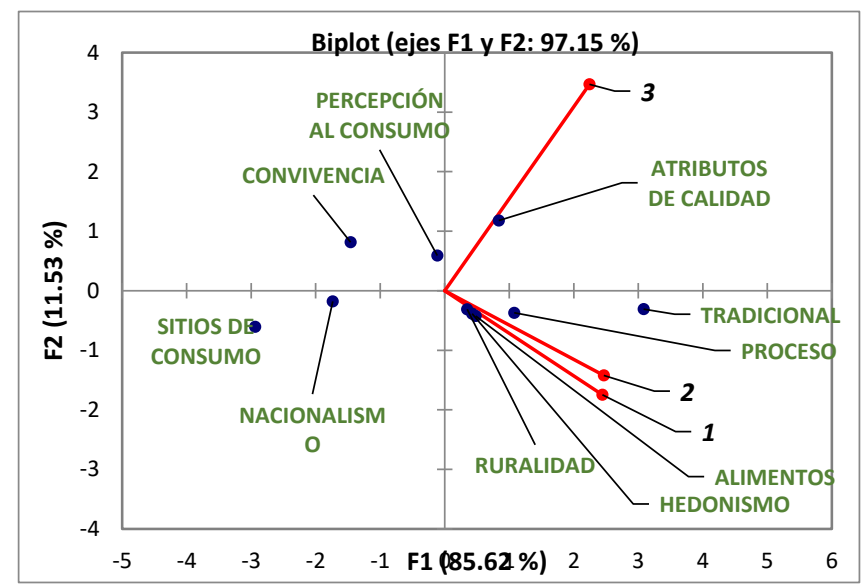

Se diferenciaron los sectores geográficos mediante la conceptualización otorgada a la palabra "pulque", particularmente en el Estado de México, donde, los significados otorgados se basan en las categorías: ruralidad, hedonismo, alimentos, proceso y tradicional. Los consumidores del Estado de México describen el pulque con categorías como ruralidad, hedonismo, alimentos, proceso y tradicionalismo; y la disposición vectorial muestra el comportamiento opuesto a las categorías "percepción al consumo" y "convivencia". Los consumidores de otros estados de la República describen el pulque mediante la categoría atributos de calidad; mostrando una evocación opuesta a las categorías "sitios de consumo" y "nacionalismo".

La frecuencia de consumo tiende a disminuir conforme aumenta la cantidad de veces de consumo en menor cantidad de tiempo; esto corrobora la hipótesis acerca de que el pulque es una bebida de consumo ocasional. La cantidad de pulque que se consume es de 1 a 2 litros; el porcentaje de entrevistados que consumen de 1 a 2 litros por ocasión fueron $43.2 \%, 59.6 \%$ y $53.7 \%$, para las zonas 1, 2 y 3, respectivamente. El porcentaje de consumidores que indican un gusto moderado por el pulque natural es del $54.1 \%$, y el $18.5 \%$ señalan tener un gusto extremado por esta bebida, mientras que el porcentaje promedio de consumidores que indican un gusto moderado por el pulque curado es del $50.5 \%$, y el $28.4 \%$ señalan tener un gusto extremado por esta bebida. Los consumidores indicaron que el precio de compra del pulque natural oscila entre $\$ 15.00$ MXN y $\$ 20.00 \mathrm{MXN}$ por litro; el $74.5 \%$ de los consumidores indicaron que el recipiente en el cual consumen el pulque es de barro.

El $64.5 \%$ de los consumidores indicaron tener mayor disposición a pagar un excedente si en el pulque se ve reflejado alguna característica que genere valor agregado; al analizar el porcentaje de excedente que los consumidores están dispuestos a pagar, se observaron diferencias significativas con el 5\% de sobreprecio (mínimo propuesto) resaltando que el mayor porcentaje se encuentra en los conceptos que no se consideran tradicionales: producto pasteurizado, enlatado y con contenido declarado bajo NOM 142 (Cuadro 3).

Cuadro 3. Porcentaje de consumidores que están dispuestos a pagar un excedente.

\begin{tabular}{l|cccccc}
\hline \multicolumn{1}{c|}{ Característica } & $\begin{array}{c}\text { Disposición } \\
\text { general }\end{array}$ & $\mathbf{5 \%}$ & $\mathbf{1 0 \%}$ & $\mathbf{2 0 \%}$ & $\mathbf{3 0 \%}$ & $\mathbf{5 0 \%}$ o más \\
\hline Artesanal y genuino & $63 \mathrm{a}$ & $17 \mathrm{ab}$ & $31 \mathrm{a}$ & $26 \mathrm{a}$ & $17 \mathrm{a}$ & $9 \mathrm{a}$ \\
Denominación de origen & $\mathbf{6 6} \mathbf{a}$ & $14 \mathrm{ab}$ & $30 \mathrm{a}$ & $27 \mathrm{a}$ & $18 \mathrm{a}$ & $11 \mathrm{a}$ \\
Producido por pequeños & $\mathbf{6 6} \mathrm{a}$ & $10 \mathrm{a}$ & $28 \mathrm{a}$ & $25 \mathrm{a}$ & $23 \mathrm{a}$ & $14 \mathrm{a}$ \\
productores & $65 \mathrm{a}$ & $22 \mathrm{ab}$ & $28 \mathrm{a}$ & $25 \mathrm{a}$ & $16 \mathrm{a}$ & $9 \mathrm{a}$ \\
Garantía de inocuidad & $66 \mathrm{a}$ & $21 \mathrm{ab}$ & $29 \mathrm{a}$ & $21 \mathrm{a}$ & $19 \mathrm{a}$ & $10 \mathrm{a}$ \\
Producción orgánica & & &
\end{tabular}




\begin{tabular}{l|cccccc}
$\begin{array}{l}\text { Declaración de contenido } \\
\text { funcional }\end{array}$ & $65 \mathrm{a}$ & $18 \mathrm{ab}$ & $25 \mathrm{a}$ & $29 \mathrm{a}$ & $17 \mathrm{a}$ & $11 \mathrm{a}$ \\
$\begin{array}{l}\text { Producción amigable con el } \\
\text { ambiente }\end{array}$ & $\mathbf{6 6 ~ a}$ & $17 \mathrm{ab}$ & $23 \mathrm{a}$ & $27 \mathrm{a}$ & $19 \mathrm{a}$ & $14 \mathrm{a}$ \\
$\begin{array}{l}\text { Pasteurizado } \\
\text { Sin aditivos añadidos }\end{array}$ & $62 \mathrm{a}$ & $36 \mathrm{bc}$ & $27 \mathrm{a}$ & $21 \mathrm{a}$ & $11 \mathrm{a}$ & $5 \mathrm{a}$ \\
$\begin{array}{l}\text { Producto enlatado } \\
\text { Contenido declarado bajo }\end{array}$ & $64 \mathrm{a}$ & $22 \mathrm{ab}$ & $30 \mathrm{a}$ & $21 \mathrm{a}$ & $16 \mathrm{a}$ & $11 \mathrm{a}$ \\
NOM 142 & $63 \mathrm{a}$ & $60 \mathrm{c}$ & $21 \mathrm{a}$ & $12 \mathrm{a}$ & $4 \mathrm{a}$ & $3 \mathrm{a}$ \\
\hline \multicolumn{1}{c}{$\quad$ Promedio } & $\mathbf{6 4 . 5}$ & $34 \mathrm{bc}$ & $31 \mathrm{a}$ & $20 \mathrm{a}$ & $11 \mathrm{a}$ & $4 \mathrm{a}$ \\
\hline
\end{tabular}

\section{Conclusiones}

El pulque es una de las bebidas tradicionales mexicanas más emblemáticas y con las cual se identifica un numero considerable de mexicanos, sin embargo, este trabajo reflejo que los significados de identidad que los consumidores le otorgan a esta bebida son bajos, asociado a la mala difusión y reputación que la industria cervecera ha construido durante décadas alrededor de esta bebida. Existe un grupo de consumidores, alrededor del $30 \%$ que valoran la bebida y están dispuestos a pagar un sobreprecio principalmente sí se consideran atributos de calidad, inocuidad que brindan mayor seguridad al consumidor.

\section{Bibliografía}

Guerrero L; Pieniak Z; Verboke W. (2009). Asociación entre el consumo tradicional de alimentos y los motivos para la elección de alimentos en seis países europeos.Revista Science Direct. Volumen 53. Núm. 1. https://www.sciencedirect.com/science/article/abs/pii/S0195666309005327

Jauregui García C. Z. (2019). Carotenoides y volátiles en elotes de jalisco e identificación de atributos intangibles por sus consumidores. Tesis de Maestría. Departamento de ingeniería Agroindustrial Chapingo.

Jodelet, D. (1989). Les représantations sociales. París, Presses Universitaires.

NORMA Oficial Mexicana NOM-142-SSA1/SCFI-2014, Bebidas alcohólicas. Especificaciones sanitarias. Etiquetado sanitario y comercial.

NMX-V-037-1972. Pulque manejado a granel. 